\title{
Adolescentes no CAPSi: relações sociais e contextos de inserção*
}

\section{Adolescents in CAPSi: social relations and integration contexts}

\author{
Amanda Dourado Souza Akahosi Fernandes', \\ Thelma Simões Matsukura²
}

http://dx.doi.org/10.11606/issn.2238-6149.v26i2p216-224

Fernandes ADSA, Matsukura TS. Adolescentes no CAPSi: relações sociais e contextos de inserção. Rev Ter Ocup Univ São Paulo. 2015 maio-ago.;26(2):216-24.

RESUMO: O objetivo do estudo foi compreender, sob a perspectiva de adolescentes usuários do CAPSi e de membros de sua rede de suporte social, os contextos de inserção e as relações sociais presentes no dia a dia desses jovens. Participaram treze adolescentes entre 12 e 18 anos e quinze membros de sua rede de suporte social. O estudo foi realizado em um CAPSi, localizado em uma cidade de grande porte no interior do Estado de São Paulo. Para coleta de dados utilizou-se dois formulários, um para a caracterização do serviço e outro para registro de dados pessoais; dois roteiros de entrevistas semiestruturadas, um aplicado com os adolescentes e outro com os membros da rede de suporte social. Para a análise dos dados adotou-se a técnica do Discurso do Sujeito Coletivo. Os resultados apontam para a desvinculação da escola, limitação das atividades de lazer e relações sociais praticamente restritas ao contexto do CAPSi. Considera-se que o estudo acrescenta ao focalizar sobre as relações sociais e os contextos de inserção a partir do reconhecimento do dia a dia dos adolescentes em sofrimento psíquico, e deve contribuir para reflexões acerca das ações junto a essa população, além de fornecer elementos para as formulações de políticas públicas.

DESCRITORES: Saúde mental; Adolescente; Questionários; Terapia ocupacional.
Fernandes ADSA, Matsukura TS. Adolescents in CAPSi: social relations and integration contexts. Rev Ter Ocup Univ São Paulo. 2015 May-Aug.;26(2):216-24.

ABSTRACT: The aim of the study was to understand, from the perspective of adolescent users of CAPSi and members of their social support network, the integration contexts and social relations present in the daily life of these youngsters. Thirteen adolescents between 12 and 18 years and fifteen members of their social support network participated in the study. The study was conducted in a CAPSi located in a large city in the countryside of state of São Paulo. To collect data two questionnaires were used, one for the characterization of the service and one for personal data registration, and two scripts of semi-structured interviews, one applied with the adolescents and one with members of the network of social support. To analyze the data the technique of Collective Subject Discourse was adopted. The results point to the disaffiliation of school, limiting leisure activities and social relations practically restricted to CAPSi context. It is considered that the study adds knowledge because it focuses on social relations and the integration contexts from the recognition of the routine of the adolescents in psychological distress and should contribute to reflections about the actions of this population, besides providing elements for the formulations of public policies.

KEYWORDS: Mental health; Adolescent; Questionnaires; Occupational Therapy.

\footnotetext{
* O estudo é parte da dissertação de mestrado da primeira autora, realizado sob a orientação da segunda autora, junto ao Programa de PósGraduação em Terapia Ocupacional da Universidade de São Carlos (PPGTO - UFSCar), o qual contou com auxílio financeiro da FAPESP. Foram apresentados trabalhos referentes à dissertação nos seguintes eventos: V Congresso Internacional de Saúde da Criança e do Adolescente; São Caetano do Sul; 24 a 27 out. de 2013; XIII Congresso Brasileiro de Terapia Ocupacional; Florianópolis; 13 a 16 out. de 2013.

1. Terapeuta ocupacional. Mestre em terapia ocupacional pelo Programa de Pós-Graduação em Terapia Ocupacional da UFSCar. Atua como professora Assistente A do Departamento de Terapia Ocupacional da UFSCar.

2. Pós-doutora pela Faculdade de Saúde Pública da USP; atua como professora adjunta do Departamento de Terapia Ocupacional da UFSCar e como docente do Programa de Pós-Graduação em Terapia Ocupacional e Programa de Pós-Graduação em Educação Especial, ambos da UFSCar.
}

Endereço para correspondência: amanda.d.fernandes@hotmail.com 


\section{INTRODUÇÃO}

$\mathrm{D}$ e acordo com o Censo Demográfico brasileiro de 2010, no Brasil há um total de 190.755.799 habitantes; destes, 24.033.747 são adolescentes entre 12-18 ${ }^{1}$. Considerando a parcela da população de crianças e adolescentes e questões relativas a sua saúde mental, a literatura aponta que as taxas de prevalência de transtornos mentais giram em torno de $10-20 \%{ }^{2}$.

Compreende-se a relevância da atenção à saúde mental durante a adolescência, uma vez que intervenções voltadas para esses indivíduos podem ser fator de extrema importância também para a vida adulta, visto que essa fase é um período de desenvolvimento e de construção de projetos $^{3}$.

Não obstante, a literatura aponta que o Brasil, assim como a maioria dos países do mundo, ainda não possui uma rede de assistência adequada à demanda existente, na medida em que a infraestrutura atual da rede pública de serviços no país não está preparada para atender em tempo hábil os casos que necessitam de atenção especializada. Além disso, os poucos serviços existentes possuem longas filas de espera e nem sempre as crianças recebem assistência adequada ${ }^{4,5}$.

Reforçando tais considerações, a Organização Mundial de Saúde indica que o Brasil precisa expandir a assistência em saúde mental para crianças e adolescentes, uma vez que o percentual de dessas em tratamento é muito baixo em todos os tipos de serviços de saúde mental, ainda mais se comparado à prevalência de transtornos psiquiátricos nessa população ${ }^{6}$.

Alguns estudos nacionais voltados para saúde mental infantojuvenil têm sido produzidos, sendo que inicialmente o enfoque predominante dos mesmos foi o epidemiológico e da busca pela caracterização dessa população quando vinculada aos serviços públicos existentes ${ }^{5,7,8}$.

Outros estudos focalizam a assistência à saúde mental infantojuvenil sob a perspectiva das políticas públicas do setor e buscam também compreender o funcionamento e as práticas desenvolvidas nos serviços. Nessas pesquisas, o CAPSi tem sido adotado como cenário de referência das políticas e de cuidado em saúde no âmbito do Sistema Único de Saúde , 10-12. $^{9}$.

Falavina e Cerqueira ${ }^{9}$ objetivaram analisar o perfil de usuários infanto-juvenis e sua trajetória no acesso aos serviços de saúde em um Centro de Atenção
Psicossocial Infantojuvenil (CAPSi) na cidade de São Paulo. Participaram da pesquisa pais ou responsáveis por crianças e adolescentes de 6 a 14 anos que frequentam o Centro. Temas como escolarização, isolamento social, discriminação, relações familiares e barreiras na obtenção dos serviços de saúde foram identificados durante a pesquisa. Os autores discutem a importância da identificação de tais problemas e apontam para a necessidade de inclusão social, melhoria e ampliação dos serviços de saúde mental para a clientela infantojuvenil e a necessidade de desmistificação da doença mental na família e no ambiente escolar. Além disso, ressaltam que crianças e adolescentes são sujeitos com necessidades particulares, de forma que compreender as especificidades dessa população usuária dos serviços de saúde mental infantojuvenil deve constituir a base do planejamento de políticas públicas.

A literatura aponta para a necessidade de mais investigações nesse campo, de forma a compreender melhor as especificidades e complexidade nele presentes, por exemplo, os desafios para a inclusão social e a efetivação de práticas de cuidado, com destaque para a articulação com a rede de assistência e para ações intersetoriais ${ }^{13-15}$.

Verifica-se também que a maior parte dos estudos nacionais que focalizam a saúde mental infantojuvenil e os CAPSi é realizada tendo como fonte de informação os prontuários, a equipe técnica e, em menor número, as famílias dessas crianças e adolescentes ${ }^{15,16}$. Não obstante, tanto as políticas públicas recentes quanto alguns estudos têm apontado sobre a importância de focalizar e dar voz aos próprios usuários, como participantes ativos da pesquisa e agenciadores de seu cuidado $^{17-19}$.

Rhoden $^{17}$, ao abordar sobre o processo de dar voz aos sujeitos da pesquisa, principalmente na infância e adolescência, ressalta que, ao inseri-los, admite-se e concretiza-se a compreensão de que são sujeitos plenos de conhecimento e autenticidade.

Assim, este estudo pretende abordar algumas questões a partir da ótica dos próprios adolescentes e de sua rede se suporte social, por exemplo: como se dá a inserção social e as relações sociais de crianças e adolescentes em sofrimento psíquico em diferentes contextos? O cuidado desenvolvido nos CAPSi responde às demandas de inserção social e as dificuldades existentes no âmbito das relações sociais? 


\section{OBJETIVOS}

O objetivo geral do estudo foi compreender, sob a perspectiva de adolescentes usuários do CAPSi e de membros de sua rede de suporte social ${ }^{*}$, os contextos ${ }^{* *}$ de inserção e as relações sociais presentes no dia a dia desses jovens.

Quanto ao objetivo específico, consiste em compreender, sob a ótica de adolescentes usuários e membros de sua rede de suporte social, o alcance do CAPSi no cuidado oferecido aos adolescentes.

\section{MÉTODO}

Este estudo integra pesquisa desenvolvida no Programa de Pós-Graduação em Terapia Ocupacional da Universidade Federal de São Carlos, sob o título "Cotidiano de adolescentes vinculados a um Centro de Atenção Psicossocial Infantojuvenil (CAPSi): realidade e perspectivas".

De acordo com os objetivos propostos, o estudo é exploratório e adota abordagem qualitativa.

\section{Participantes}

Participaram do estudo treze adolescentes com idade entre 12 e 18 anos, inseridos no CAPSi. Quanto aos critérios de seleção, além da faixa etária, os adolescentes deveriam estar inseridos no CAPSi há pelo menos três meses, não ter como indicação de queixa principal o uso abusivo de substâncias psicoativas e ter condições de estabelecer um diálogo, segundo avaliação dos profissionais do serviço. Além dos adolescentes, participaram do estudo quinze membros da rede de suporte social dos usuários, indicados pelos próprios jovens durante a entrevista.

\section{Local da pesquisa: Centro de Atenção Psicossocial Infantojuvenil}

O estudo foi realizado em um Centro de Atenção Psicossocial Infantojuvenil (CAPSi) destinado a crianças e adolescentes, localizado em uma cidade de grande porte no interior do Estado de São Paulo. Esse serviço é referência no cuidado à saúde mental infantojuvenil na região, conta com equipe completa e funciona há mais de dez anos em período integral. A escolha do local ocorreu por conveniência e respeitando-se alguns critérios que buscaram garantir que os cuidados oferecidos não fossem atravessados pelo viés de situações decorrentes de equipamentos recém-introduzidos e/ou com equipe incompleta.

\section{Instrumentos para coleta de dados}

- Formulário para registro de dados pessoais dos adolescentes: composto por dezenove questões que buscaram caracterizar os participantes, como idade, escolaridade e situação escolar, estrutura e composição familiar, dentre outras.

- Formulário de caracterização do serviço: a partir de vinte questões, abordou a estrutura do serviço, funcionamento e estratégias de cuidado.

- Roteiro de entrevista semiestruturada aplicado junto aos adolescentes: composto por 29 questões que abordaram o dia a dia no CAPSi e em outros contextos, relações sociais, redes de suporte social, dentre outras.

- Roteiro de entrevista semiestruturada aplicado junto aos membros da rede suporte social dos adolescentes: composto por onze questões que abordaram a percepção do adolescente e seu dia a dia, as expectativas e demandas relativas ao cuidado oferecido e outras considerações acerca da realidade vivenciada pelo adolescente em seus diferentes contextos de inserção.

\section{PROCEDIMENTOS}

\section{Aspectos éticos}

O estudo foi aprovado pelo Comitê de Ética em Pesquisa em Seres Humanos da Universidade Federal de São Carlos (UFSCar) sob o parecer número 112.514, no dia 11 de setembro de 2012. Além disso, foi solicitada autorização da Secretaria Municipal de Saúde do município ao qual o CAPSi pertence e do próprio serviço. Com a aprovação dos mesmos, o desenvolvimento do estudo foi iniciado.

\footnotetext{
* Vaux ${ }^{20}$ ressalta que o suporte social deve ser considerado como um metaconstruto com três componentes conceituais distintos, ou seja, recursos da rede de suporte, comportamento suportivo e as avaliações subjetivas de suporte, ou o suporte social percebido. de suporte, ou o suporte social percebido.

** O conceito de contexto adotado neste estudo se apoia na teoria Bioecológica de Bronfrenbrenner ${ }^{21}$. Compreende-se o contexto a partir de quatro níveis ambientais - microssistema, mesossistema, exossistema e macrossistema, onde as atividades, papéis sociais e relações interpessoais e inter-relações acontecem, assim como as ideologias, valores e crenças, culturas e subculturas estão presentes.
} 


\section{Coleta de dados}

A coleta de dados foi realizada entre dezembro de 2012 a maio de 2013 e, após o aceite do CAPSi para a realização do estudo, o coordenador do serviço respondeu à ficha de caracterização, e um cronograma para inserção do pesquisador no serviço foi estabelecido. Assim, o contato com os adolescentes foi efetivado por meio do acompanhamento e da participação da pesquisadora nas diferentes atividades do serviço, ocasião em que a pesquisa foi explicada aos adolescentes e o convite para a participação foi efetivado. Dentre aqueles que aceitaram participar, foi também solicitado aos responsáveis a autorização quanto ao envolvimento dos adolescentes.

Todas as entrevistas foram realizadas no CAPSi; durante a entrevista com os adolescentes, foram identificados quais outros atores e contextos estavam envolvidos no cotidiano dos mesmos, por meio de questões presentes no roteiro de entrevista. Assim, para a composição dos participantes que representariam a rede de suporte social do adolescente, uma questão específica solicitava ao adolescente que indicasse pessoas que o conhecesse bem, de que ele gostasse e que fizessem parte de sua rede de suporte social. A partir dessa identificação estabeleceu-se os principais atores, que, em concordância com o adolescente, foram convidados a responder ao roteiro de entrevista direcionado aos mesmos. Ressalta-se que todas as pessoas da rede de suporte social indicadas pelo adolescente aceitaram participar da pesquisa.

\section{Análise de dados}

Os dados advindos dos formulários foram organizados e analisados descritivamente.

Os dados advindos das entrevistas foram examinados utilizando-se a técnica do Discurso do Sujeito Coletivo - DSC ${ }^{22}$. O objetivo da técnica do DSC, pautada na Teoria das Representações Sociais, é que, por meio de um único discurso, a opinião de uma coletividade seja dada, porém sem descaracterizar a natureza qualitativa de cada depoimento analisado ${ }^{22}$.

\section{RESULTADOS}

Os resultados serão apresentados em duas etapas; primeiramente os relativos à caracterização do serviço e dos participantes, seguidos dos resultados advindos das entrevistas.

\section{a) Caracterização do serviço}

Em relação à área de abrangência, o CAPSi contemplava 36 Unidades Básicas de Saúde (UBS) dessas regiões. Além disso, havia um total de 1.346 prontuários registrados até dezembro de 2012, sendo que, destes, 99 eram de adolescentes em tratamento.

Em relação às atividades desenvolvidas no CAPSi existiam diferentes tipos, e dentre as trinta atividades elencadas, essas estavam distribuídas em: atendimentos individuais e familiares, grupos variados, reuniões de equipe, grupo de pais, oficinas, passeios, acolhimento, dentre outras.

Nos grupos, participam diferentes profissionais do serviço; alguns eram abertos, outros fechados. A maioria dos grupos acontecia no próprio serviço, enquanto outros, em algumas ocasiões, ocorriam em contextos diferentes (p.ex. grupo de esportes).

\section{b) Caracterização dos participantes}

Apresenta-se no Quadro 1 a seguir a caracterização dos adolescentes participantes da pesquisa.

Observa-se, a partir das informações apresentadas, que, dos treze membros, oito são meninos, apenas seis frequentam a escola, sete já reprovaram de ano e apenas cinco moram com ambos os pais.

Apresenta-se no Quadro 2 a caracterização dos membros da rede de suporte social dos adolescentes. Observa-se, a partir das informações apresentadas, que, dentre os participantes indicados como pertencentes à rede de suporte dos adolescentes, doze dos quinze membros são profissionais do CAPSi.

\section{c) Os adolescentes usuários do CAPSi: relações sociais e contextos de inserção}

A seguir serão apresentados os resultados advindos das entrevistas com os adolescentes e com os membros da rede de suporte social. Os DSCs apresentados são acompanhados da letra A, quando se referem a discursos produzidos a partir da análise das entrevistas com os adolescentes, e com a letra R, os DSCs advindos das entrevistas com os membros da rede de suporte social.

Ao falarem sobre as relações familiares, os adolescentes revelaram que, ao mesmo tempo em que reconhecem aspectos positivos da família e das relações dela advindas, também apontam para muitos conflitos, violência e rompimentos que envolvem todo o núcleo, como é possível verificar nos DSCs a seguir. 
"Legal. Quando a minha mãe vai lá, a gente brinca, vai visitá minha tia, sai, conversa, passeia, a gente vai no shopping, vai no bosque, na casa dos parentes e amigos. Eu e a minha mãe também, às vezes, vamos para o shopping, convida meu pai, a gente vai uma hora e volta só as seis. Ah, meu pai, quando tem lição para fazer meu pai me ajuda." (A).

"Minha família é muito grande e complicada. Só tem briga, meu pai, eu e minha mãe e minha irmã também, família inteira. Meu avô também me xinga muito, então a minha mãe fala pra eu ficar na minha vó, ele fala assim, não, você não vai ficar não, ai ele pega e xinga a minha vó. Bom também teve uma época que eu estava meio doidinho ai eu comecei a xingar todo mundo, xingava minha mãe e meu pai, queria que eles saíssem de casa, ai eles falavam pode sair, vai na casa da sua tia, vai na sua colega, ai eu fiquei saindo um monte de vezes, ai depois eles ficaram com raiva de mim, ficaram me xingando, e meu pai também, mas aí depois melhorou." (A).

Quadro 1 - Caracterização dos adolescentes da pesquisa

\begin{tabular}{|c|c|c|c|c|c|c|}
\hline & & & Caracte & ção dos adolesc & ites & \\
\hline Adolescente & Sexo & Idade & Frequenta a escola & Escolaridade & Repetiu de ano & Reside com \\
\hline A & $\mathrm{M}$ & 18 & Não & $6^{\circ}$ ano & Sim & Irmão/Rua \\
\hline B & M & 13 & Sim & $5^{\circ}$ ano & Sim & Avó \\
\hline $\mathrm{C}$ & $\mathrm{F}$ & 16 & Sim & $2^{\circ}$ ano do EM & Não & Avó e avô \\
\hline $\mathrm{D}$ & M & 13 & Sim & $6^{\circ}$ ano & Não & Pais, irmã e avó \\
\hline $\mathrm{E}$ & M & 17 & Não & $1^{\circ}$ ano do EM & Sim & Pais e irmã \\
\hline $\mathrm{F}$ & M & 18 & Não & $8^{\circ}$ ano & Sim & Mãe \\
\hline G & M & 14 & Sim & $7^{\circ}$ ano & Sim & Mãe, irmã e irmão \\
\hline $\mathrm{H}$ & $\mathrm{F}$ & 14 & Não & $9^{\circ}$ ano & Não & Pais, 2 irmãos, 2 tios, tia, 2 primos \\
\hline I & M & 17 & Sim & $3^{\circ}$ ano do EM & Não & Pais e irmã \\
\hline $\mathrm{J}$ & $\mathrm{F}$ & 12 & Não & $2^{\mathrm{a}}$ série & Sim & Mãe, pai e irmão \\
\hline $\mathrm{K}$ & M & 17 & Não & $9^{\mathrm{a}}$ ano & Sim & Avô e avó \\
\hline $\mathrm{L}$ & $\mathrm{F}$ & 12 & Não & $6^{\circ}$ ano & Não & Avós, tios, primos \\
\hline M & $\mathrm{F}$ & 14 & Sim & $9^{\circ}$ ano & Não & Avós, irmão, primo \\
\hline
\end{tabular}

Fonte: Elaborado pela autora

Quadro 2 - Caracterização dos membros da rede de suporte social dos adolescentes

\begin{tabular}{|c|c|c|l|}
\hline \multicolumn{2}{|c|}{} & \multicolumn{2}{|c|}{ Caracterização da rede de suporte social } \\
\hline Rede do adolescente & Sexo & Idade & Relação com adolescente \\
A & M & 27 & Profissional CAPS \\
B & F & 37 & Profissional CAPS \\
C & F & 43 & Profissional CAPS \\
D & M & 27 & Profissional CAPS \\
E & F & 34 & Profissional CAPS \\
F & F & 46 & Profissional CAPS \\
G & F & 43 & Profissional CAPS \\
H & F & 52 & Profissional CAPS \\
I & M & 17 & Usuário do CAPS \\
& F & 48 & Profissional CAPS \\
J & F & 33 & Mãe \\
& F & 43 & Profissional CAPS \\
K & M & 24 & Profissional do CAPS \\
L & F & 32 & \\
\hline
\end{tabular}

Fonte: Elaborado pelo autora 
Os adolescentes também relataram sobre seus relacionamentos com amigos. Nesses relatos identificou-se que tais relacionamentos têm origem no próprio CAPSi, na igreja que frequentam e alguns poucos na escola. Também se revelou que as amizades envolviam desde uma troca mais afetiva e constante até o simples compartilhar de um mesmo contexto, como ilustram os DSCs a seguir.

"Tenho (amigos) daqui, os daqui do CAPSi... tudo daqui. A gente conversa." (A).

"Tenho mais amigos, amigos é só da igreja. Na igreja a gente canta, se interage demais." (A).

Sob outra perspectiva, mas ainda sobre os relacionamentos sociais, foi solicitado aos participantes que representaram a rede de suporte social dos adolescentes que relatassem sobre o relacionamento que possuem com esses. Os DSCs a seguir expressam sobre este resultado.

\begin{abstract}
"Minha relação com ele é entre amor e ódio, então quando estou concordando com o que ele fala, quando escuto, está tudo bem. Às vezes quando não posso ele briga comigo, fica de mal e tudo mais. Tem dia que o bicho pega, porque ele está numa idade dificil. Mas não é também assim, de 1 a 100 é 20\% que ele é assim violento, de bater boca, de agressão física." (R).

"Eu acho que a minha relação com ele mesmo é a questão do trabalho, uma relação paciente-funcionário, mas ao mesmo tempo tenho um carinho por ele claro... eu sinto que a minha relação com ele é uma relação bem afetuosa, eu tenho muito afeto por ele. Ele confia bastante, ele consegue, eu sei que tem algumas coisas que pra ele ainda é difícil dizer, mas ele consegue, tem uma abertura. Agora ele está conseguindo ter um lugar, um porto seguro." (R).
\end{abstract}

Em relação aos contextos de inserção dos adolescentes, este tema foi abordado ao longo de doze questões que envolveram, por exemplo, o contexto escolar, o próprio CAPSi e outros.

Sobre a inserção no Centro, tanto os adolescentes quanto os membros da rede de suporte social relataram sobre as mudanças no dia a dia quando o adolescente começou a frequentar o serviço. Além disso, os participantes também apresentaram demandas e sugestões, como pode ser verificado nos DSCs, a seguir.

"Tudo (mudou). Me deu mais ânimo. Sei lá, eu era muito quietinho no canto, não falava nada, ficava lá fazendo lição, com vontade de chorar, eu não aguentava pressão na escola, ai eu vim pra cá melhorou, eu não fico tão quietinho, essas coisas." (A).

"Bom, eu queria que (o CAPSi) me ajudasse a... sei lá é, bom, fazer mais coisas, mais atividades, aqui fora, em outros lugares, tipo assim me divertir, que nem ir no parque aquático. Tipo sair mais com os amigos, entendeu?” (A).

"Está ajudando justamente nesse processo de interação, para facilitar a interação social, eu acho que facilita a vida dele lá fora. No grupo ele gosta de vir porque ele fica com o pessoal do grupo, tem contato com os adolescentes daqui, como ele tinha lá fora também, mas com uma visão diferente, ajuda a dar outro foco." (R).

Sobre o contexto escolar, importa ressaltar que a maior parte dos adolescentes encontra-se desvinculada da escola. Dentre os motivos apontados, tem-se desde dificuldades em acompanhar os conteúdos formais até a impossibilidade de permanecer na escola em função do sofrimento psíquico (choro incontrolável, alucinações etc.).

Já dentre os adolescentes que frequentam o contexto escolar, as referências a esse espaço ficam focalizadas no relato sobre o próprio comportamento, como expressam os seguintes DSCs.

"Os meus amigos até que são meio ruins, porque falam besteira para um amigo meu, eles falam assim sua mãe, sua irmã. A última vez que ele falô da minha irmã eu joguei água nele, com a garrafa de água. Falei que era a última vez que falava dela." (A) .

"Eu faço besteira, de primeiro eu jogava cadeira, mesa na escola, jogava um monte de cadeira, só ouvia barulho... agora eu só jogo às vezes quando eu fico muito nervoso. Fica tudo no chão as cadeiras." (A).

\section{DISCUSSÃO}

Em relação ao contexto familiar, os resultados indicaram a presença de conflitos familiares, dificuldades de relacionamento, estrutura familiar muitas vezes desorganizada e conturbada, marcada pela violência, fragmentação e conflitos. Entretanto, apesar das dificuldades familiares, os adolescentes realizam atividades juntamente com a família, sendo que alguns familiares também são citados como rede de suporte social.

Para a reflexão desses resultados, é pertinente utilizar os conceitos e estudos que tratam sobre fatores e mecanismos de risco e proteção à saúde mental ${ }^{23,24}$. Assim, é possível que a violência, desestruturação familiar, dentre 
outros, compreendidos como fatores de risco, implicam sofrimento psíquico dos adolescentes, e, da mesma forma, o afeto, os relacionamentos suportivos, dentre outros fatores, podem ser protetivos frente aos riscos ${ }^{25}$.

Ainda sobre a rede de suporte social, este estudo revela o papel que ocupa o trabalhador de saúde mental do CAPSi no dia a dia desses jovens. O fato de os participantes, em sua maioria, indicarem profissionais do CAPSi como membros de sua rede de suporte social pode ser compreendido como um diferencial qualitativo nas relações e práticas ali estabelecidas, e também, conforme analisa Salles $^{26}$, pode sugerir que as redes sociais dos usuários os tornam pertencentes ao sistema social e ao contexto que os permeiam.

Não obstante, é possível que a rede de suporte social composta em sua maioria por profissionais do CAPSi reforce a compreensão de que esse equipamento é um dos raros, senão únicos, contextos de inserção desses jovens, nos quais relacionamentos mais efetivos se concretizam. Fontes ${ }^{27}$, ao abordar o apoio social no campo da saúde mental, coloca que este implica incorporar o campo institucional (os profissionais de saúde e suas instituições), porque nesses espaços passa a acontecer parte significativa do cotidiano dessas pessoas, seu campo de sociabilidades secundárias e o de seus cuidadores; e também os campos de sociabilidade primária dos doentes mentais: amigos, parentes, colegas de trabalho, vizinhos, ou seja, pessoas com quem se relaciona e de quem retira apoio para o enfrentamento de seu sofrimento psíquico.

Quanto às propostas existentes no CAPSi focalizado na presente pesquisa, há uma gama de atividades que visam à inserção dos usuários. Essas permeiam desde a convivência, os atendimentos individuais, grupos e oficinas dentro do Centro, até as intervenções externas que ocorrem em locais públicos do município. Identificou-se neste estudo, por meio dos discursos dos participantes, que tais práticas são realizadas na tentativa de que esses espaços se tornem dispositivos para que os processos de inclusão social e de estabelecimento de relações aconteçam, sendo possível reconhecer alguns ganhos e avanços nesses âmbitos.

Ainda que as portarias regulatórias do Ministério da Saúde apontem o CAPSi como dispositivo que visa às práticas de reabilitação psicossocial e também da clínica ampliada, preconizando ações que extrapolem os muros e facilitem a entrada desses adolescentes na comunidade e a inclusão e relações sociais ${ }^{28}$, verifica-se que a maior parte das ações ocorre internamente e há o desafio de ampliá-las para outros contextos além do próprio.
No que se refere ao contexto escolar, neste estudo identificou-se que esse espaço de convivência e aprendizado não fazia parte da vida da maior parte dos adolescentes participantes. Estudos recentes, ainda que raros, têm se debruçado a focalizar sobre a escola e a saúde mental de crianças e adolescentes e podem fornecer elementos para a compreensão do processo de desvinculação do contexto escolar observado neste estudo ${ }^{29}$. Por exemplo, o estudo de Matsukura et al. ${ }^{30}$ identificou que crianças com dificuldades emocionais se sentiam tristes na escola e se referiam a esse âmbito de maneira negativa, como sendo chato ou ruim.

Acredita-se que a doença e a saúde mental em sua complexidade tragam consigo desafios e demandas que a escola, ainda que sob o paradigma da inclusão, não tem tido sucesso. Indica-se que estudos que se debrucem sobre as possibilidades e limites acerca da efetivação e condução de ações intersetoriais, com destaque para a escola, devem ser colocados na pauta da agenda de pesquisadores do campo.

Além disso, é preciso que políticas públicas sejam efetivadas, de forma a garantir os direitos dessa população a frequentar a escola regular, assim como garantir a qualidade do ensino e possibilidades de aprendizado. Compreende-se que este caminho viabilizará não só o aprendizado, como também possibilidades de inserção social em outros contextos, de forma a assegurar melhores perspectivas de vida, no que se refere à sociabilização, profissionalização e projetos de vida.

\section{CONSIDERAÇÕES FINAIS}

Este estudo objetivou dar voz a adolescentes usuários do CAPSi e compreender seus contextos de inserção e suas relações sociais. Dentre os resultados revelou-se que a rede de suporte social desses indivíduos é composta pelos trabalhadores do próprio CAPSi e que este equipamento é o contexto central de inserção de tais jovens.

Discutiu-se, a partir da perspectiva colocada pelos participantes do estudo, acerca da necessidade de criação de espaços e serviços que ampliem as possibilidades de inclusão e relações sociais, onde as subjetividades estejam implicadas, na direção da garantia de direitos. Assim, considera-se que, para além da viabilização das políticas públicas existentes, a demanda pela ampliação das mesmas é prioridade.

Importa destacar que os limites deste estudo devem ser considerados, como o limite dos contextos aqui abordados, e indica-se a necessidade de continuidade e aprofundamento de estudos nesse campo. 


\section{REFERÊNCIAS}

1. Instituto Brasileiro de Geografia e Estatística. População residente, por situação do domicílio e sexo, segundo os grupos de idade [citado 23 jul. 2012]. Disponível em: ftp://ftp.ibge.gov.br/Censos/Censo_Demografico_2010/ Caracteristicas_Gerais_Religiao_De ficiencia/tab1_1.pdf.

2. Organização Mundial da Saúde, Organização PanAmericana de Saúde. Relatório sobre a saúde no mundo: saúde mental: nova concepção, nova esperança. Genebra: World Health Report; 2001.

3. Mângia EF, Assumpção CN, Quinta JM, Rufino MF. Necessidades de adolescentes com sofrimento psíquico. Rev Ter Ocup Univ São Paulo. 2003;14(3):123-32. DOI: http://dx.doi.org/10.11606/issn.2238-6149.v14i3p123-132.

4. Fleitlich B. A saúde nos jovens brasileiros: quais são os principais problemas e como podemos ajudar? J Bras Psiquiatria. 2005;54(3):168-9.

5. Paula CS, Duarte CS, Bordin IS. Prevalence of mental health problems in children and adolescents from the outskirtS of São Paulo city: treatment needs and service capacity evaluation. Rev Bras Psiquiatr. 2007;29(1):11-7. DOI: http://dx.doi.org/10.1590/S1516-44462006005000012.

6. Who-Aims. Report on Mental Health System in Brazil, WHO and Ministry of Health. Brasília; 2007.

7. Paula CS, Vedovato MS, Bordin IAS, Barros MGSM, D'Antino MLF, Marcadante MT. Mental health and violence among sixth grade students from a city I the state of São Paulo. Rev Saúde Pública. 2008;42(3):524-8. DOI: http://dx.doi.org/10.1590/S0034-89102008005000027

8. Anselmi L, Fleitlich-Bilyk B, Menezes AM, Araújo CL, Rohde LA. Prevalence of psychiatric desorders in Brasilian birth cohort of 11 yearolds. Soc Psychiatr Epidemiol. 2009;45(1):135-42. DOI: 10.1007/s00127-009-0052-2.

9. Falavina OP, Cerqueira MB. Saúde mental infanto-juvenil: usuários e suas trajetórias de acesso aos serviços de saúde. Rev Espaço Saúde. 2008;10(1):34-46. Disponível em: http://www.uel.br/ccs/espacoparasaude/v10n1/Artigo $\% 20$ 5\%20-\%20referente\%20ao\%2070-2008.pdf.

10. Oliveira MFAPB. O trabalho oculto na rede de atenção psicossocial a crianças e jovens em sofrimento psíquico [Dissertação]. São Paulo: Faculdade de Saúde Publica, Universidade de São Paulo; 2010.

11. Beltrame MM. Infância e saúde mental: reflexões sobre a dinâmica de trabalho de um CAPSi [Dissertação]. Maringá: Universidade Estadual de Maringá; 2010.

12. Tano BL. Os centros de atenção psicossocial infantojuvenis (CAPSi) e as práticas de cuidado para as crianças e adolescentes em intenso sofrimento psíquico;
[Dissertação]. São Carlos: Universidade Federal de São Carlos; 2014.

13. Delfine PSS, Bastos ENE, Gois CWL, Tofoli FLO. Apoio matricial em saúde mental na atenção primária à saúde: uma análise da produção científica e documental. Interface. 2013;17(45):287-300. DOI: http://dx.doi.org/10.1590/ S1414-32832013005000012.

14. Ramires VRR, Benetti SPC, Silva FJL, Flores GG. Saúde mental de crianças no Brasil: uma revisão de literatura. Interação Psicol. 2009;13(2):311-22. DOI: http://dx.doi. org/10.5380/psi.v13i2.7601.

15. Santos PL. Problemas de saúde mental de crianças e adolescentes atendidos em um serviço público de psicologia infantil. Psicol Estudo. 2006;11(2):315-21. DOI: http:// dx.doi.org/10.1590/S1413-73722006000200010.

16. 16. Borsa JC, Souza DS, BandeiraDR. Prevalência dos problemas de comportamento em uma amostra de crianças do Rio Grande do Sul. Psicol Teoria Prática. 2011;13(2):15-29.

17. 17. Rhoden S. A pesquisa com crianças: a criança como sujeito de pesquisa. Semin Nac Arte Educ. 2012;(23):4107. Disponível em: http://seer.fundarte.rs.gov.br/index.php/ Anaissem/article/view/112/205.

18. Catão VMC. Criança: sujeito de história e cultura: princípios metodológicos da pesquisa com crianças. Rev Uniabeu. 2012;5(9):188-212. Disponível em: http://www.uniabeu. edu.br/publica/index.php/RU/article/view/371/pdf_148

19. Brasil. Ministério da Saúde. Conselho Nacional do Ministério Público. Atenção psicossocial a crianças e adolescentes no SUS: tecendo redes para garantir direitos. Brasília: Ministério da Saúde; 2014. p.60. Disponível em: http://bvsms.saude.gov.br/bvs/publicacoes/atencao_ psicossocial_criancas_adolescentes_sus.pdf.

20. Vaux A. An ecological approach to understanding and facilitating social support. J Social Personal Relationships. 1990;7(4):507-18. DOI:10.1177/0265407590074007.

21. Bronfenbrenner U. A ecologia do desenvolvimento humano: experimentos naturais e planejados. Porto Alegre: ArtMed; 1996.

22. Lefèvre F, Lefèvre AMC. Pesquisa de representação social: um enfoque qualiquantitativo, a metodologia do discurso do sujeito coletivo. Brasília: Liber Livro; 2010.

23. Assis SG, Avancis JQ, Oliveira RVC. Socioeconomic inequalities and child mental health. Rev Saúde Públ. 2009;43(Supl 1):92-100. Available from: http://www. scielosp.org/scielo.php?script=sci_arttext\&pid=S0034$89102009000800014 \& \operatorname{lng}=$ en\&nrm=iso. 
24. Rutter M. Psychosocial resilience and protective mechanisms. Am J Orthopsychiatry. 1987;57(3):316-31. DOI: 10.1111/j.1939-0025.1987.tb03541.

25. Greenberg MT, Domitrovich C, Bumbarger B. The prevention of mental disorders in school-aged children: current state of the field. Prevent Treat. 2001;4(1):1-59. Available from: http://prevention.psu.edu/pubs/documents/ mentaldisordersfullreport.pdf.

26. Salles MM. Vida cotidiana de usuários de CAPS: a (in) visibilidade no território [Tese]. São Paulo: Escola de Enfermagem, Universidade de São Paulo; 2011.

27. Fontes BASM. Redes sociais e saúde: sobre a formação de redes de apoio social no cotidiano de portadores de transtorno mental: política \& trabalho. Rev Cien Soc (João Pessoa). 2007;(26): 87-104. Disponível em: http://www.nucleodecidadania.org/ nucleo/extra/2007_07_16_06_10_51.pdf.

Recebido para publicação: 06.01.15

Aceito para publicação: 03.06.15
28. Brasil. Ministério da Saúde. Portal da Saúde. Infanto-Juvenil. Disponível saúde mental no SUS: os centros de atenção psicossocial [citado mar. 2012]. Disponível em: http://portal.saude.gov.br/portal/saude/visualizar_texto. cfm?idtxt=30452\&janela $=1$.

29. D'Abreu LCF, Marturano EM. Identificação de problemas de saúde mental associados à queixa escolar segundo o DAWBA. Psico. 2011;42(2):152-8. Disponível em: http:// revistaseletronicas.pucrs.br/ojs/index.php/revistapsico/ article/view/8487.

30. Matsukura TS, Fernandes ADSA, Cid MFB. Fatores de risco e proteção à saúde mental infantil: o contexto familiar. Rev Ter Ocup Univer São Paulo. 2012;23(2): 122-9. DOI: http://dx.doi.org/10.11606/issn.2238-6149. v23i2p122-129. 